\title{
Proliferative nodule in melanocytic nevi mimicking deep penetrating nevus*
}

\author{
Ana Almodovar-Real ${ }^{1}$ \\ Jose Aneiros-Fernandez ${ }^{3}$
}

\author{
Alejandro Molina-Leyva ${ }^{2}$ \\ Miguel Antonio Diaz-Martinez ${ }^{1}$
}

DOI: http:/ / dx.doi.org/10.1590/abd1806-4841.20175383

\begin{abstract}
Proliferative nodules can occasionally arise on congenital and acquired melanocytic nevi. At first sight their clinical and pathologic features cause alert to both dermatologist and dermatopathologist. However, proliferative nodules are typically benign, regression is common and there is minimum risk of malignization. We present a new case of proliferative nodule in melanocytic nevi with features of deep penetrating nevus.
\end{abstract}

Keywords: Diagnosis; Nevus, pigmented; Pathology

\section{INTRODUCTION}

The histological interpretation of proliferative nodules (PNs) arising on congenital or acquired nevi can represent a diagnostic challenge for pathologists. The presence of atypical histological features is not uncommon, making their distinction from melanoma problematic. ${ }^{1}$

\section{CASE REPORT}

A 29-year-old woman followed-up in the unit of pigmentary lesions due to multiple melanocytic nevi, showed changes in a nevus on the left scapular region during dermoscopic examination. A new polychromatic nodule not present in the previous dermoscopy was observed after one year (Figure 1). Given the clinical suspicion of melanoma the lesion was completely excised. Histopathology examination revealed a large and well-circumscribed nodule with cells gathered in small and well-formed uniform nests. Mitotic activity was almost null. Large pale cells with hyperchromatic nuclei, vacuolated cytoplasm, and dusty-appearing melanin were present (Figure 2, 3A and 3B). Melan A was positive and HMB- 45 was negative (Figures $4 \mathrm{~A}$ and $4 \mathrm{~B}$ ). Ki67 was less than 1\% (Figure 5). The patient has been in good health and there has been no sign of recurrence after 2 years of follow-up.

\section{DISCUSSION}

Proliferative nodules (PNs) can occasionally arise in congenital and acquired melanocytic nevi. They typically appear in young children, but congenital cases and cases in adults have been reported..$^{2-4}$ Clinically, PNs are presented as papules, nodules, or plaques that can protrude or be situated deeply into the nevus. The changes in nevi morphology produced by PNs can be subtle making difficult

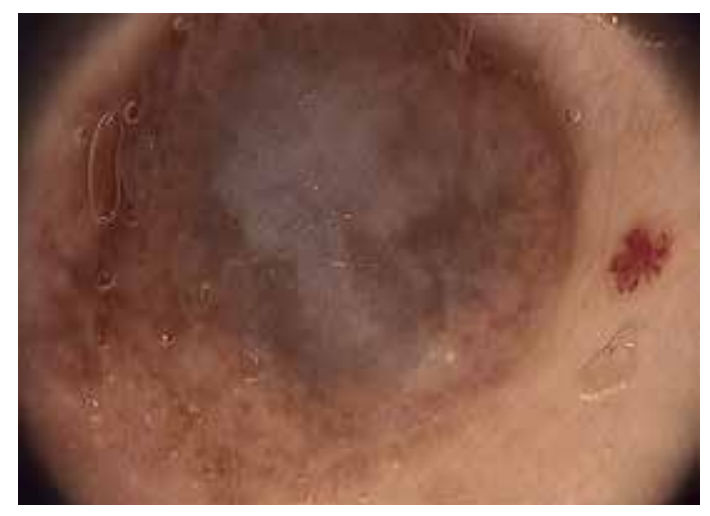

FIGURE 1: Dermoscopy shows a polychromatic nodule in compound nevus

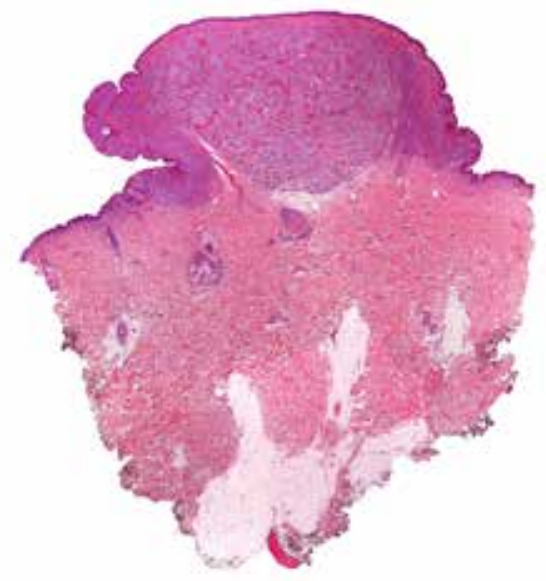

Figure 2: Well-circumscribed nodule. HE 10x

\footnotetext{
Received on 19.11.2015

Approved by the Advisory Board and accepted for publication on 21.01.2016

Study conducted at the University Hospital - Granada, Spain.

Financial support: none.

Conflict of interest: none.

Department of Dermatology, San Cecilio University Hospital - Granada, Spain.

Department of Dermatology, Torrecárdenas Hospital - Almería, Spain.

Department of Pathology, San Cecilio University Hospital - Granada, Spain.

○2017 by Anais Brasileiros de Dermatologia
} 
to identify them in a bare eye examination, thus dermoscopic examination and follow-up has a key value to diagnose this entity. ${ }^{2,3}$ Their etiology is unclear; it is hypothesized that they are a monoclonal

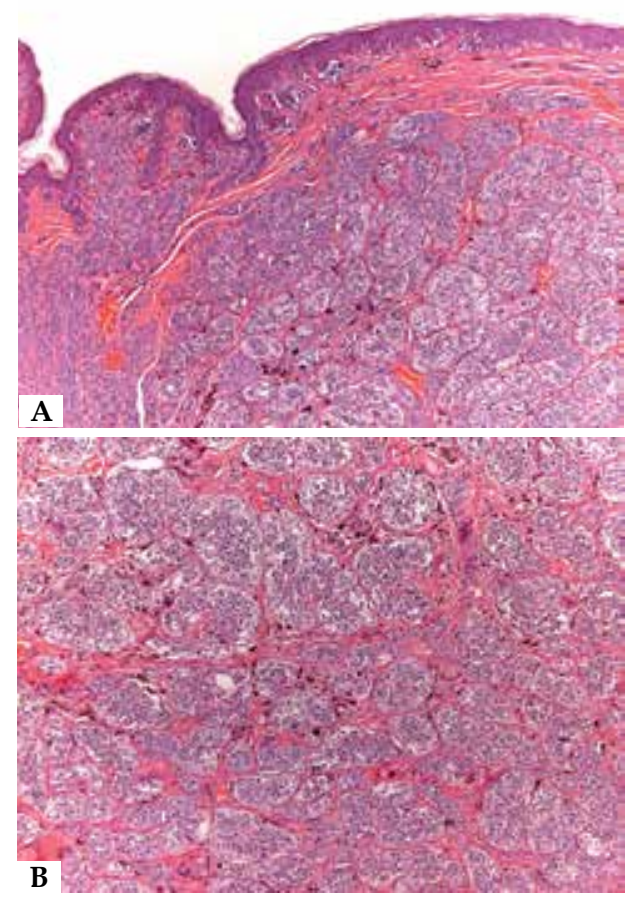

Figure 3: A. Cells in small, well-formed nests that are approximately of the same size and shape. HE 100x. B. Cells in small, well-formed nests that are approximately of the same size and shape. HE 200x
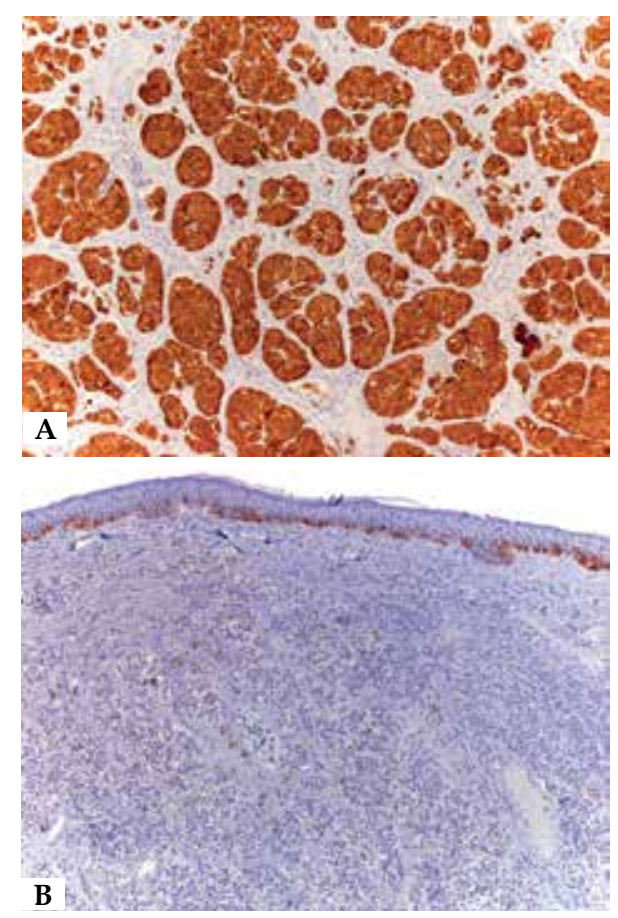

Figure 4: A. Melan A positive in PN; 200x. B. HMB- 45 negative in $\mathrm{PN}$; $100 \mathrm{x}$

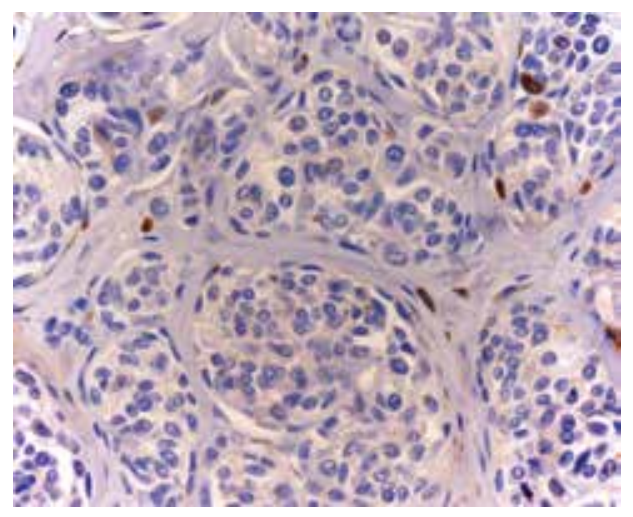

Figure 5: Ki67<1 \% in PN; 400x

proliferation of melanocytic cells. ${ }^{4}$ Generally PNs exhibit a darker pigmentation compared with the nevus of origin and usually have the same texture. On their natural history, a progressive reduction on consistency, texture, and pigmentation is observed. ${ }^{5}$

PNs can show diverse histological patterns including features of well-known types of nevi, like cellular nevus, blue nevus, Spitz nevus, forms with neural or other mesenchymal differentiation or deep penetrating nevus. ${ }^{5}$ Like in our case-report, intensely pigmented PNs developing in a congenital or an acquired melanocytic nevus have a close morphological resemblance with a deep penetrating nevus. Deep penetrating nevi are benign lesions in which fascicles of oval melanocytes exhibit deep infiltration into the dermis. Their histological pattern can be quite alarming, showing cells with vesicular or hyperchromatic nuclei in the center of which a large eosinophilic nucleolus might be present. In spite of these findings, the distinction from melanoma can be made based on the sharp circumscription, the vertical orientation and symmetry of the lesion, the scarcity of mitoses, the presence of small nests of cells rather than sheets of cells, and the absence of necrosis. The regularity in the distribution of the pigment contained in melanophages, evenly scattered throughout the lesion in a chessboard pattern, also confirms the benignity of the lesions. ${ }^{5}$

Typically, the most helpful features to diagnose PNs are the presence of maturation, low mitotic rate, low-grade cytological atypia, absence of necrosis and absence of inflammatory infiltrate. However, it is important to keep in mind that in a small subset of cases, benign PNs can be large, highly cellular and may have atypical melanocytes, mitoses, zones of necrosis and even ulceration. ${ }^{6}$

We present a new case of $\mathrm{PN}$ in acquired melanocytic nevi with deep penetrating nevus-like features. This entity can clinically and histologically be confused with a malignant transformation. Therefore, a thorough histological evaluation is needed to rule out the presence of a melanoma. $\square$ 


\section{REFERENCES}

1. Phadke PA, Rakheja D, Le LP, Selim MA, Kapur P, Davis A, et al. Proliferative nodules arising within congenital melanocytic nevi: a histologic, immunohistochemical, and molecular analyses of 43 cases. Am J Surg Pathol. 2011;35:656-69.

2. Kiyohara T, Sawai T, Kumakiri M. Proliferative nodule in small congenital melanocytic naevus after childhood. Acta Derm Venereol. 2012;92:96-7.

3. Leech SN, Bell H, Leonard N, Jones SL, Geurin D, McKee PH, et al. Neonatal giant congenital nevi with proliferative nodules: a clinicopathologic study and literature review of neonatal melanoma. Arch Dermatol. 2004;140:83-8.

4. Lowes MA, Norris D, Whitfeld M. Benign melanocytic proliferative nodule within a congenital naevus. Australas J Dermatol. 2000;41:109-11.

5. Guido M, LeBoit PE. Proliferative nodules in congenital and acquired nevi. In: Guido M, LeBoit PE, editors. Histological diagnosis of Nevi and Melanoma 2nd ed. City: Springer Science \& Business Media; 2014. p. 97-112.

6. Feito-Rodríguez M, de Lucas-Laguna R, Bastian BC, Leboit P, González-Beato MJ, López-Gutiérrez JC, et al. Nodular lesions arising in a large congenital melanocytic naevus in a newborn with eruptive disseminated Spitz naevi. $\mathrm{Br} \mathrm{J}$ Dermatol. 2011;165:1138-42.

\author{
MAILING ADDRESS: \\ Ana Almodovar-Real \\ Department of Dermatology, \\ San Cecilio University Hospital \\ Avenida Doctor Olóriz 16 \\ ES-18012 Granada, Spain \\ Email - anamariaalmodovar@gmail.com
}

How to cite this article: Almodovar-Real A, Molina-Leyva A, Aneiros-Fernandez J, Diaz-Martinez MA. Proliferative nodule in melanocytic nevi mimicking deep penetrating nevus. An Bras Dermatol. 2017;92(2):231-3. 